\title{
The Research Wheeled Armored Vehicles Engine Overheating Fault Diagnosis
}

\author{
Yi Yao ${ }^{1, a}$, Baiyuan Gu ${ }^{2, b}$, Meng Zhang ${ }^{3, c}$, Wen Cao ${ }^{4, d}$ \\ ${ }_{1,2,3,4}$ Armor Technique Institute of PLA, Changchun ,China \\ a3174296251@qqcom, b3063543236@qq.com, ${ }^{\text {c,d } 1441789825 @ q q . c o m ~}$
}

Keywords: Wheeled Armored Vehicles; Engine; Overheating; Fault Diagnosis

\begin{abstract}
Engine overheating is a common fault of wheeled armored vehicles, and the cause of overheating is very more. In this paper, the author through their own professional knowledge and experience, finding out the reason of several engine overheating, and summarizes several useful overheating fault diagnosis methods. It has a certain technical reference value.
\end{abstract}

\section{The Performance of the Engine Overheating}

Engine overheating fault symptoms is main shown that: more than $100{ }^{\circ} \mathrm{C}$ temperature indicator, radiator steam and boiling water mouth visible phenomenon, engine working, you can accelerate metal tapping, to close the engine ignition switch can't immediately shut down, etc. And the engine cooling system protect the engine under all conditions can keep working in the appropriate temperature range, so to be sure, in the process of vehicle engine overheating, the vast majority of the original for the engine cooling system malfunction.

Engine in normal work, combustion gas temperature can be as high as $1800{ }^{\circ} \mathrm{C}$ to $2000{ }^{\circ} \mathrm{C}$, directly in the environment of high temperature friction parts (such as cylinder liner and piston, piston and piston rings, valve and valve seat, etc.) if not in time to cool, may be caused by thermal expansion, destroy the normal clearance and stuck to each other, but also led to the decrease of the viscosity of lubricating oil, lubricating oil membrane formation, aggravate wear parts, etc. The results in [1]. Conclusion engine overheat reasons, therefore, is an important theoretical base for the wheeled armored vehicles overheat breakdown, plays an important guiding significance.

\section{The Causes Analysis of Engine Overheating}

Wheeled armored vehicle engine overheating many reasons, mainly has the following kinds.

(1) the radiator radiator pipe leaks or damaged part. Due to limited water tank of water stored in water, especially after discharge pipe blockage, cannot circulating cooling water, it is easy to make the engine overheating.

(2) the thermostat damage. Due to the thermostat damage, does not automatically adjust cooling intensity, make proper circulation cooling water can't flow, the engine can't work under optimum temperature state and overheating.

(3) the fan belt too loose or skid. Fan belt skid, cooling water quantity of heat cannot be distributed through the radiator smoothly, the engine can not fully cooling and overheating.

(4) the pump performance deterioration. Mainly is the water pump seal damage, water pump drops, not mandatory for cooling cycle, the hot water in the engine to the radiator for cooling, then return to cooling water chamber.

(5) the fan blade bending deformation. And air volume reduction and cause vibration, and even destroyed the radiator core tube, will lead to engine overheating.

(6) the radiator cover to steam and inward outward - two valves work bad air. The resulting system is unable to control cooling system, cooling water boiling point in the engine can't work under normal temperature.

(7) ignition timing phrase. Ignition early to produce detonation, burning time is too late to extend, heat can't send out in time, all will lead to exhaust temperature and engine overheating. 
(8) the lack of oil, oil pan lubrication system fails, the oil pump can't effectively put the machine oil into all lubricating points, that caused by the bad lubrication, half dry friction temperature too high.

\section{The Fault Diagnosis of Engine Overheating and Screening Method}

\section{1 check the cooling water level}

The engine cooling water, water shortage, is the most direct reason for engine overheating, and there are main causes of the cooling water shortage.

(1) normal consumption. Engine in normal work, the temperature of the cooling water usually within the range of $80{ }^{\circ} \mathrm{C}$ to $95{ }^{\circ} \mathrm{C}$ [3], at this time there will be a small amount of cooling water to reduce due to evaporation. If not timely to supplement, can cause water shortages caused by the engine is overheating. Therefore, usually need to inject a certain amount of cooling water when the engine temperature is not too high, to keep the amount of water between the highest and lowest water level.

(2) to reduce leakage. Engines often caused by excessive wear pumps water seal leakage; Thus generated in the wet cylinder due to cavitation pitting or holes, and due to the effect of corrosion of the antifreeze and cause of the timing gear plate of decay, oil radiator cover soil hole corrosion, etc. Diagnosis can be the first cooling water to fill the radiator, if after long time back to the lowest level, then have leakage of the cooling system, must be timely to find the causes for the leakage, and the failure parts for repair, and then the cooling water to the normal water level.

\subsection{Check the Fan and Fan Belt}

If under the condition of the cooling water is enough, the engine still overheating phenomenon happens, you will need to inspect the fan and fan belt. A check on this, the first to see whether the fan belt skid, whether the fan rotation, if the skid, you should check the status of the fan belt itself and belt tensioning situation, conform to the provisions of the technical requirements, adjust or replace as needed. The second depends on the rotation of the fan blade. Commonly used the following two methods to diagnose the decision:

(1) cold. Vehicle after overnight, fan clutch plate and clutch plate between still remain with silicone oil, as a result of the silicone oil viscosity is very big, so when changing the fan blade by hand, should be more arduous. When starting up the engine, make them under the cold operating at medium speed for $1 \mathrm{~min} \sim 2 \mathrm{~min}$, in order to make work cavity silicone oil return oil storage chamber, then stop the engine, then dial the fan blade, reoccupy hand should be relatively easy, this is normal.

(2) the hot check. After starting the engine, make the cooling water temperature is close to $90{ }^{\circ} \mathrm{C}$ to $95{ }^{\circ} \mathrm{C}$, and then carefully listen to the noise of the engine fan place changes, if in a few minutes the noise increased, fan speed can be increased quickly, to be in when running at full speed, the engine shut down again, with toggle fan blade finally feel more arduous, it is normal. If the results don't meet the above requirements, the silicon oil clutch failure, should be replaced.

Generally in the car, mostly use the fan to control the temperature of the engine cooling water. In general, when the water temperature reached $89{ }^{\circ} \mathrm{C}$ to $92{ }^{\circ} \mathrm{C}$, electronic fan should be rotates at low speed; $97{ }^{\circ} \mathrm{C}$ to $103{ }^{\circ} \mathrm{C}$ when rotating at high speed. If you do not meet above requirement, to check whether the circuit has a problem.

\subsection{Checking and Repairing the radiator}

If the fan and fan belt is under normal operation of the engine overheating, still going to look at the radiator reasons.

(1) the appearance inspection. First look at the radiator fin is pore dust, dirt, etc. Some sundry jam, if blocked, will cause the cooling performance is bad, causes the engine overheating. At this point, can be in the back of the radiator with compressed air or injection pressure tap water to remove impurities, and then the residual dirt on the radiator with brush clean.

In addition, due to mechanical damage, make fin deformation and attached together, also is another factor that makes the poor performance of radiator radiator. Therefore, can be used stalloy 
will heat sink, sufficiently, each attached with a recovery before the deformation condition. For some of the vehicles in front of the radiator use blinds (or wind curtain), if the shutter (or the wind curtain) can't normal open also causes engine overheat, at this time to find out the specific fault parts out in a timely manner.

(2) internal inspection. First, check the cooling pipe blockage. If the radiator cooling pipe blockage, will reduce the cooling performance, also is the main factor that make the engine overheating. Whether the radiator cooling pipe stuck, after warming up, with the hand touch the upper and lower two parts of the radiator, pay attention to the difference in temperature, under normal circumstances, the temperature difference between the upper and lower needs to have certain but not too much. If feel the temperature difference is particularly marked, suggests that the radiator cooling pipe is blocked, it will be carried out on the radiator cooling water pipe cleaning, eliminate congestion.

In addition, check the thermostat. Normal thermostat, should open at $68{ }^{\circ} \mathrm{C}$ to $72{ }^{\circ} \mathrm{C}, 80{ }^{\circ} \mathrm{C}$ to $85{ }^{\circ} \mathrm{C}$ should be fully open. If open and fully open temperature is higher than the above provisions, the channel in the cooling water temperature is too high, the engine is overheating.

Check the thermostat's method: the thermostat down, then with the thermometer in the vessel with hot water at the same time, and then gradually heating, observe the thermostat valves are open and completely open when the temperature is in accordance with the specified value. If the thermostat is damaged, it should be replaced.

\section{4 check the ignition timing}

Ignition advance Angle (gasoline engine) or fuel injection advance Angle (diesel) lead or lag, are likely to cause the engine overheating, because no matter the ignition advance Angle (or injection) lead or lag, burning, burning gas and the area of the cylinder wall contact will be bigger than design, so the heat to the cooling water will increase, causing abnormally rise the temperature of the engine, the fuel consumption at the same time also will increase. Therefore, should check the ignition timing (or injection), make it conform to the technical requirements.

\section{Conclusion}

High temperature in the engine work is not to be despised, any kind of small fault will cause major accidents to the engine. Familiar with the engine running state, in a timely manner to eliminate the problems arising from the work, to guarantee the engine working in good condition.

\section{References}

[1] Xinyan Zhai,Xinyong Zhang. Engine overheating fault examination and ruled out[J] . Shandong agricultural mechanization, 2001, 13: 22.

[2] Patrick S. Numerical simulation of electric power steering (EPS) system[J].KOYO Engineering Journal:English Edition,2002,(16):52-56.

[3] Willett P K,Kirubarajan T. Proceedings of SPIE[J].Orlando:SPIE,2002,(01).

[4]Rao C R,Pathak P K,Koltchinskii VL.[J].Journal of Statistical Planning and Inference,1997,(02):257.

[5] Zhao Wanzhong,Shi Guobiao,Lin Yi. Road feeling of electric power steering system based on mixed control[J].Journal of Mechanical

Engineering,2009,(04):142-146.doi:10.3901/JME.2009.04.142. 